\title{
Quasi-static and dynamic deformation of an in-situ Ti-based metallic glass composite in supercooled liquid region
}

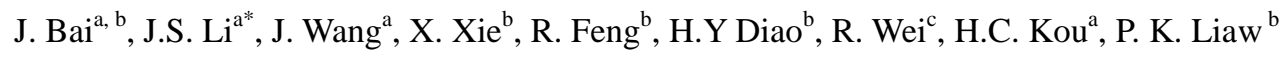 \\ a. State Key Laboratory of Solidification Processing, Northwestern Polytechnical University, Xi'an \\ 710072, China \\ b. Department of Materials Science and Engineering, The University of Tennessee, Knoxville, TN \\ 37996-2200, USA \\ c. School of Materials Science and Engineering, Zhengzhou University, Zhengzhou 450001, China
}

\begin{abstract}
The quasi-static and dynamic deformation behaviors of the Ti-based bulk metallic glass (BMG) composite $\left(\mathrm{Ti}_{48} \mathrm{Zr}_{20} \mathrm{Nb}_{12} \mathrm{Cu}_{5} \mathrm{Be}_{15}\right)$ were investigated in the supercooled liquid region. The present BMG composite exhibits homogenous viscous flow under quasi-static deformation, but shear-band dominated inhomogeneous plastic deformation upon dynamic loading. With the increase of the temperature, the flow stress decreases in both quasi-static and dynamic conditions. Due to the effective plastic accommodation of dendrites to the glass matrix, characteristic of the formation of shear steps and multiple dislocations, the remarkable plasticity under dynamic deformation has been obtained in the present BMG composite in SLR. The constitutive equation for the present BMG composite upon quasi-static loading is obtained. Upon dynamic loading, the flow stress approximately follows a linear relationship with the temperature: $\sigma(\mathrm{MPa}) \approx 2,791-2.11 T(\mathrm{~K})$.
\end{abstract}

Keywords: Composite materials; Metallic glasses; Mechanical properties; Strain; Supercooled liquid region.

\footnotetext{
*Corresponding author. Tel.:+86 298849 1074; Fax: +86 2988460294.

E-mail address: ljsh@nwpu.edu.cn.
} 


\section{Introduction}

The excellent properties of bulk metallic glasses (BMGs), such as high strength, high hardness and good net-shaping ability in supercooled liquid region (SLR), have rendered them as promising candidates of engineering materials [1-3]. However, the ambient brittleness caused by the highly-localized shear deformation in BMGs has greatly limited their widespread applications [4-6]. In recent years, design of in-situ BMG composites provides an effective way to alleviate the brittleness [7-9]. By precipitating crystalline second phase during solidification, the localized deformation has been greatly mitigated by the formation of multiple shear bands in the glass matrix [9-11]. Up to now, the enhanced plasticity has been obtained in some Ti- [12-15], $\mathrm{Zr}-[9,16], \mathrm{Cu}-$ [17] based $\mathrm{BMG}$ composite, etc. at ambient temperature.

The mechanical properties of these in-situ BMG composites are mostly studied at room temperature. However, the response of these in-situ BMG composites to some extreme conditions, such as at cryogenic [18, 19], high temperatures [20-22] and upon dynamic loading [23-25], is very important to understand their mechanism and broaden application. The deformation behavior of in-situ BMG composites under quasi-static deformation in SLR has been reported in few studies. The introduction of the dendritic second phase has shown significant influence on the high-temperature flow of the glass matrix. Fu et al. claimed that the homogenous deformation of in-situ La-based BMG composite with a high-volume fraction of second phases is dominated by the second phase in SLR, resulting in the derivation from the transition state model for monolithic BMGs [26]. Qiao et al. further reported that the high volume of second phase $(\sim 43 \%)$ in the Zr-based BMG composite has greatly retarded the softening of the glass matrix by enhancing the viscosity, offering up to a tensile stenghth of $970 \mathrm{MPa}$ even in SLR [20]. Zhang et al. [27] found that viscous flow of the Cu-Zr based BMG in SLR could be no longer entirely explained in term of viscous flow of the glass matrix after precipitating a high volume of nanocrystallines. Thus, the existence of high-volume second phase in BMG composites plays a significant role on the viscous flow of the glass matrix. However, up to now, deformation behavior of these in-situ BMG composites with a high volume fraction of second phase in SLR still lacks fully understanding. Moreover, the dynamic deformation of BMG composites in SLR is scarcely reported. Detailed investigation will not only contribute to understanding their deformation mechanism, but also promoting their potential application. Based on these regards, the deformation behavior of in-situ Ti-based BMG composite under both quasi-static and dynamic loading was investigated in the 
SLR and their micromechanisms were clarified.

\section{Material and methods}

Master ingots of Ti-based BMG composites with a nominal composition of $\mathrm{Ti}_{48} \mathrm{Zr}_{20} \mathrm{Nb}_{12} \mathrm{Cu}_{5} \mathrm{Be}_{15}$ in atomic percent were fabricated by arc melting pure elements of $\mathrm{Ti}, \mathrm{Zr}, \mathrm{Cu}, \mathrm{Nb}(>99.99$ wt.\%) and $\mathrm{Be}(>$ 99.95 wt.\%). Cylindrical samples with $50 \mathrm{~mm}$ in length and $3 \mathrm{~mm}$ in diameter are obtained by the copper mould casting methods. The thermal properties of the present BMG composites were determined using the differential scanning calorimetry (DSC, Pekin-Elmer DSC 7) at the heating rate of $20 \mathrm{~K} / \mathrm{min}$. The glass transition temperature, $T_{\mathrm{g}}$, and the crystalline temperature, $T_{\mathrm{x}}$, of the present BMG composite were determined to be $653 \mathrm{~K}$ and $723 \mathrm{~K}$, respectively. The phase compositions of the current BMG composites were examined by X-ray diffraction (XRD, Bruker D2 Phaser). Rod samples with a diameter of $3 \mathrm{~mm}$ and an aspect ratio of $1: 2$ were cut and well polished for the quasi-static tests performed on a MTS 810 materials testing machine with strain rates from $2 \times 10^{-4} / \mathrm{s}$ to $1 \times 10^{-2} / \mathrm{s}$ within SLR $(653 \mathrm{~K}, 683 \mathrm{~K}$, and $713 \mathrm{~K})$ and the temperature was carefully controlled within $\pm 1 \mathrm{~K}$. Dynamic deformation tests were conducted on the Split Hopkinson Pressure Bar (SHPB) with a furnace from $653 \mathrm{~K}$ to $713 \mathrm{~K}$ at the strain rate of $1.3 \times 10^{3} / \mathrm{s}$. The microstructure evolution and fracture surface were identified by the scanning-electron microscope (SEM, VEGA3 TESCAN). High-resolution transmission-electron microscope (HRTEM, Tecnai G2 F30) was employed to further identify the structure evolution after deformation.

\section{Results}

\subsection{Microstructure and DSC analysis}

Fig. 1(a) exhibits the microstructure of the present BMG composite. The dendritic second phase (white contrast) is homogenously distributed in the continuous matrix (dark contrast). The volume fraction of the second phase is estimated to about $52 \% \pm 2 \%$ by image analysis. The X-ray diffraction pattern of crystal peaks overlaying on the broad scattering peak in Fig. 1(b) further indicates that the second phase is body-centered cubic $\beta$-Ti solution and glass matrix is in an amorphous status.

3.2 Mechanical properties

\subsubsection{Quasi-static compressive deformation}

Fig. 2 is the true stress versus true strain curves of the present BMG composites at different strain rates within the temperatures from $663 \mathrm{~K}$ to $713 \mathrm{~K}$, respectively. It shows that whatever the strain rate is, the steady-state flow can be quickly reached at the temperature of both $663 \mathrm{~K}$ and $683 \mathrm{~K}$, revealing 
that the homogenous deformation occurs in the SLR. However, at $713 \mathrm{~K}$, although the steady flow state can reach at relatively higher strain rate $\left(5 \times 10^{-3} / \mathrm{s}-1 \times 10^{-2} / \mathrm{s}\right)$, obvious work hardening is observed at the lower strain rates.

To understand the variation of stresses with the strain rates and temperature, the double logarithmic plots of the steady-state flow stress versus strain rates at different temperatures is shown in Fig. 3. Here, the initial steady-state flow stress stage was chosen for the strain rate of $2 \times 10^{-4} / \mathrm{s}$ and $5 \times$ $10^{-4} / \mathrm{s}$, because of the work hardening. With the increase of strain rates, the flow stress increases at all temperatures. The strain-rate sensitivity, $m$, can be calculated by $m=\partial \sigma_{T} / \partial \dot{\varepsilon}_{T}\left(\sigma_{T}, \dot{\varepsilon}_{T}\right.$ are the flow stress and strain rates at a certain temperature respectively). Thus, as displayed in Fig. 3, the strain-rate sensitivity factors are all much lower than the unit, e.g., at $663 \mathrm{~K}, m$ falls in the range of 0.07 - 0.21, which indicate that the non-Newtonian flow dominates the flow behavior of the present BMG composite in SLR. Furthermore, the strain-rate sensitivity increases slightly from a value of $0.07-0.21$ to $0.09-0.35$ when temperature increases from $663 \mathrm{~K}$ to $713 \mathrm{~K}$. Although they are still less than a unit, the slightly increased $m$ implies that the high temperature contributes to the trend of the Newtown flow of the present BMG composites. Compared with the monolithic BMGs which own the larger $m$ in SLR at high temperatures or low strain rates, the low strain-rate sensitivity factors $m$ in the current in-situ BMG composites further indicates that the high volume dendrites have dramatically retarded viscous flow of the glass matrix in SLR.

\subsubsection{Dynamic compressive deformation}

Fig. 4(a) shows the true stress versus true strain curves upon dynamic loading at a constant strain rate of $1.3 \times 10^{3} / \mathrm{s}$. Up dynamic loading, the present BMG composite fractures after a remarkable plastic flow in the SLR. At $663 \mathrm{~K}$, the present BMG composite has a high strength of $1391 \mathrm{MPa}$ along with the plasticity of $9.5 \%$. As the temperature increases, the flow stress reduced to $1288 \mathrm{MPa}$ at $713 \mathrm{~K}$. In previous study, the brittle fracture has been reported in $\mathrm{Zr}$-based monolithic BMGs at high strain rates in SLR [28]. Therefore, the high strength and plasticity of the present in-situ BMG composites under dynamic loading in SLR could contribute to their potential applications. The relationships between stresses, strains and temperatures upon dynamic loading are given in Fig. 4(b). As the rising of temperature, both the strength of the glass matrix and crystalline phase are expected to be downward. Thus, according to the rule of mixture, it's reasonable to understand the decrease of the flow stress of BMG composites also decreases with the temperature increases. 


\subsection{Fracture morphology}

The samples after quasi-static $\left(2 \times 10^{-4} / \mathrm{s}\right)$ and dynamic $\left(1.3 \times 10^{3} / \mathrm{s}\right)$ deformation at $683 \mathrm{~K}$ are exhibited in Fig. 5. As shown in Fig. 5(a), the drum-like sample is obtained after quasi-static deformation, corresponding to the large viscous flow. In order to observe the microstructure evolution, the sample was then cut in half. As shown in Fig. 5(b), the dendrites aligned perpendicular to applied stress after deformation, and no voids are formed near the interfaces between the matrix and dendrites, demonstrating the strong atomic bonding and good load transfer between the dendrites and glass matrix. However, upon dynamic loading, shear failure fracture can be observed in Fig. 5(c). The magnified image of the lateral surface in the inset reveals the formation of multiplication of shear bands in SLR. This trend signifies that the transition from the viscous homogenous flow at quasi-static loading in SLR to shear-band dominated inhomogeneous deformation at high strain rates. Fig. 5(d) displays the fracture surface after dynamic loading, which displays that melting layer covers on the surface, indicative of the great temperature increase in the critical shear bands.

\section{Discussion}

As mentioned above, strain hardening can be seen in the later deformation stage at the lower strain rate of $2 \times 10^{-4} / \mathrm{s}$ and $5 \times 10^{-4} / \mathrm{s}$, as shown in Fig. 2. Similar phenomena are also been observed in some Ti based [29, 30], Zr- based [31] BMGs, which are considered to be related to the structural change of the amorphous phase caused by the long-time deformation at high temperature. To identify the microstructure evolution, the DSC curves of samples after deformation at $2 \times 10^{-4} / \mathrm{s}$ at $663 \mathrm{~K}$ and $713 \mathrm{~K}$ are shown in Fig. 6, respectively. Compared to that at $663 \mathrm{~K}$, the obvious diminution of exothermal entropy from $36.91 \mathrm{~J} / \mathrm{g}$ to $21.79 \mathrm{~J} / \mathrm{g}$ at $713 \mathrm{~K}$, reveals the nanocrystallization of glass matrix during deformation. Kim et al. [31] and Bletry et al. [32] claimed that the occurrence of nanocrystallization during deformation can increase the back stress of BMGs in SLR, which causes the strain hardening of BMGs. During deformation, the high temperature and low strain rate are in favor of the crystallization in SLR. Thus, the formation of nanocrystals in the glass matrix during deformation at higher temperature could result in additional "composite effects" in the present BMG composite, which further leads to the strain hardening of the present BMG composite.

For the thermal-activated deformation of metallic materials, deformation behavior is influenced by both the strain rate, $\dot{\varepsilon}$, and deformation temperature, $T$. An empirical hyperbolic-sine equation proposed by Sellars and Tegart [33] to describe the rheological behavior at high temperature has been 
successfully employed in crystalline materials and metallic glasses [34]. The effects of temperature and strain rate on the materials are usually expressed as,

$$
\dot{\varepsilon}=A[\sinh (\alpha \sigma)]^{n} \exp \left(-\frac{Q}{R T}\right)
$$

where $A$ and $\alpha$ are materials constants, which are independent of the temperature. $n$ is a stress exponent. $\sigma$ corresponds to the flow stress at certain temperature, $T$, and strain rate, $\dot{\varepsilon}$. Here, $\sigma$ is taken as steady-stae flow stress in the current study. $Q$ is the activated energy and $R=8.3145$ is the gas constant. $\alpha$ can be determined by $\alpha=\frac{\partial \ln \dot{\varepsilon}}{\partial \sigma} / \frac{\partial \ln \dot{\varepsilon}}{\partial \ln \sigma}$ [35]. Thus, $\alpha=0.00287$ can be obtained for the present BMG composite.

Then, taking the logarithm of Eq. 1, linear relationship can be obtained as Eq. 2

$$
\ln \dot{\varepsilon}=\ln A+n \ln [\sinh (\alpha \sigma)]-\frac{Q}{R T}
$$

As shown in Fig, 7(a), which gives the plots of $\ln \dot{\varepsilon}-\ln [\sinh (\alpha \sigma)]$, the $A$ and $n$ can be obtained by the average value of intercepts and slopes at different temperatures, respectively. In addition, at a certain strain rate, Eq. 2 could be transformed to Eq. 3 .

$$
\ln [\sinh (\alpha \sigma)]=\frac{1}{n} \ln A-\frac{1}{n} \ln \dot{\varepsilon}-\frac{Q}{n R T}
$$

By plotting the $\ln [\sinh (\alpha \sigma)]$ vs. $1 / n R T$, the slope gives the apparent activated energy of deformation, $Q$. The calculated results for the present BMG composites are summarized in Table. 1.

The validity of the Sellars-Tegart model is verified by plotting $\ln Z$ as a function of $\ln [\sinh (\alpha \sigma)]$, where $\mathrm{Z}$ is the Zener-Hollomon parameter, defined as $Z=\dot{\varepsilon} \exp (Q / R T)$. A good linear relationship between $\ln Z$ and $\ln [\sinh (\alpha \sigma)]$ in Fig. 7(b) indicates the availability of the Sellars-Tegart model in the present BMG composites. Therefore, the constitutive equation of the present composites upon the quasi-static deformation in SLR can be obtained as:

$$
\dot{\varepsilon}=1.53 \times 10^{24}[\sinh (0.00287 \sigma)]^{4.032} \exp \left(-\frac{4.37 \times 10^{5}}{8.3145 T}\right)
$$

The apparent activated energy of deformation, $\mathrm{Q}$ is obtained to be $436 \mathrm{KJ} / \mathrm{mol}(4.53 \mathrm{eV})$ for the present BMG composites, which is lower than the typical $\mathrm{Pd}_{40} \mathrm{Ni}_{10} \mathrm{Cu}_{30} \mathrm{P}_{20}(5.0 \mathrm{eV})$ [32] and some Ti-, Zr- based BMGs, for example $\mathrm{Zr}_{55} \mathrm{Al}_{10} \mathrm{Ni}_{5} \mathrm{Cu}_{30}$ (5.1 eV) [36], $\mathrm{Ti}_{41.5} \mathrm{Cu}_{37.5} \mathrm{Ni}_{7.5} \mathrm{Zr}_{2.5} \mathrm{Hf}_{5} \mathrm{Sn}_{5} \mathrm{Si}_{1}(6.3 \mathrm{eV}$ ) [30], but higher than the self diffusion activation energy of principle element Ti (2.00 eV- $3.138 \mathrm{eV})$ 
[37] in the crystalline lattice. In crystalline materials, the activated energy can be interpreted as the barrier of bulk / lattice diffusion, etc., of a certain species of atoms in the matrix [30]. The superplasticity of metallic glasses is usually considered to be related with the simultaneous migration of a group of atoms. Thus, in view of the composite structure of the present BMG composite, the mediate activated energy between metallic glass and crystalline implies that synergetic movement of the glass matrix and crystalline dendrites could contribute to the high-temperature viscous flow of the present BMG composite.

As shown in Fig. 3, the present BMG composite exhibits the low strain rate sensitivity, less than a unit in all conditions, indicative of the non-Newtonian viscous flow for the present BMG composite in SLR. The apparent viscosity, $\eta$, of the present BMG composite can be given as [27, 34]:

$$
\eta=\frac{\sigma}{3 \dot{\varepsilon}}
$$

Substituting Eq. (1) into Eq. (4), then Eq. (4) can be rewritten as:

$$
\eta=\frac{\sigma}{3 A[\sinh (\alpha \sigma)]^{n} \exp (-Q / R T)}
$$

For the present BMG composite, assuming that the Newtonian flow occurs at a lower strain rate, which corresponds to the low stresses. Then, $\sinh (\alpha \sigma) \approx \alpha \sigma$. Therefore, Eq. (5) can be reduced as,

$$
\eta_{N}=\frac{\sigma}{3 A(\alpha \sigma)^{n} \exp (-Q / R T)}
$$

where, $\eta_{\mathrm{N}}$ is the ideal viscosity in a Newtonian manner.

Then, the normalized viscosity, $\eta / \eta_{N}$, can be obtained by combining Eq. (5) and Eq. (6)

$$
\frac{\eta}{\eta_{N}}=\frac{(\alpha \sigma)^{n}}{[\sinh (\alpha \sigma)]^{n}}
$$

Fig. 9 exhibits the normalized viscosity as the function of Zener-Hollomon parameter. All the experiment data fall within the Non-Newtonian deformation and follow the master curve of viscosity given as Eq. (7). It can be seen that the non-Newtonian to Newtonian flows trend to occur at low Z values, i.e. higher temperature or lower strain rates. Whereas, the deformation corresponding to a high $\mathrm{Z}$ results in a non-Newtonian flow.

On the other hand, as far as the dynamic deformation of the present BMG composites, the shear fracture and the formation of multiple shear bands in Fig. 5(c) reveal that the macroscopic deformation 
transforms from the homogeneous viscous flow in quasi-static deformation to inhomogeneous deformation upon dynamic deformation. Meanwhile, it's worth noting that in comparison to the poor plasticity previously reported in the monolithic BMGs upon dynamic deformation [38], the present BMG composite owns remarkable plasticity and high fracture strength upon dynamic loading in SLR, indicating that the dendrites play an important role on impeding the prompt propagation of shear bands in SLR.

The dependence of flow stress on temperature and are shown in Fig. 9. Li et al [1]. studied the temperature-dependent behavior of BMGs, finding that the flow stress follows a linear relationship with increasing the temperature, i.e. the stress decreases linearly with the temperature increases. It can be seen that an approximate linear relationship also dominates in the present BMG composite in SLR, which implies that the strength of the glass matrix might exert an important role on the flow strength of BMG composites upon dynamic loading in SLR. The linear relationship between the stress and temperature can be expressed as:

$$
\sigma \approx 2,791-2.11 T(\mathrm{MPa})
$$

To better understand the micromechanism of the present BMG composite upon dynamic loading in SLR. Fig. 10(a) and (b) display the TEM and HRTEM images after dynamic deformation at $713 \mathrm{~K}$. Stepped interfaces can be observed, as shown in Fig. 10(a). These steps are considered to be formed to accommodate shear strain between the dendrites and glass matrix, indicative of the coordinative effects of dendrites during deformation [10, 12, 39]. Meanwhile, corresponding to the severe plastic deformation in dendrites, dense dislocation generates in the dendrites. The inset of Fig. 10(a) is the selected-area-electron diffraction pattern of the matrix. The concentric diffused rings indicate no crystallization occurs in the glass matrix upon dynamic loading. Fig. 10 (b) exhibits the HRTEM image of the interface between the dendrites and the matrix. After deformation, it shows a good atom-scaled bonding between the crystalline dendrites and glass matrix, which ensures the effective loading transfer between them. The insets (b1) and (b2) of Fig. 10(b) are the corresponding inverse fast Fourier transformation (IFFT) of the regions marked by the black and white rectangular regions in Fig. 10(b), respectively. As marked by "T" in (b1), multiple dislocates generate near the interface. The disordered structure in (b2) further indicates the amorphous state of the matrix after deformation.

Fig. 11 (a) summarizes the compressive stress vs. strain curves for the typical Ti- [29, 30], Zr- [38], $\mathrm{Cu}-$ [27], La- [26] Mg-[40] based BMGs and their homologous composites. Noting that the flow stress 
of their composites counterpart is commonly larger than the monolithic BMGs at the same strain rate and temperature, the main reason should lie in the introduction of second phase in composites could dramatically increase the viscosity of the glass matrix in SLR, resutling in the higher resistance to the viscous flow of the matrix [20]. On the other hand, relatively to clearly downward concavity of the stress-strain rate curve in the monolithic BMGs, all the composites exhibit subdued increasing trend of the flow stress with the increase of the strain rate at certain temperature, i.e., the lower strain-rate sensitivity of the composites in SLR. As far as the viscous flow of the composites, especially which own higher fraction of second phases, the existence of second phases is expected to modify the flow of glass matrix. For the glass matrix, it tends to the Newtown flow at high temperatures and low strain rates, i. e., $m$ trends to be a unit. However, for the second phase, for example the $\beta$-Ti dendrites in the present BMG composite, they usually have a very low value of $m(0.008-0.44)$ [41]. Thus, the concurrent mechanism of the dendrites and glass matrix will lead to the decreased strain-rate sensitivity [42]. Fig. 11(b) shows the dynamic mechanical properties of the present BMG composites as well as some reported monolithic BMGs [28, 38, 43] and composites [43] in SLR. It's worth noting that the present BMG composites possess remarkable plasticity and high stress in comparison to the monolithic BMGs in SLR. In monolithic BMGs, in absence of the effective barrier to the propagation of shear bands, once the critical shear bands generate, it's prone to develop and mature into shear cracks due to the severe diabatic shear deformation in shear bands. However, for the present BMG composite, the dendrites are still considered to effectively accomodate the inhomogenous deformation and hinder the propagation of shear bands in the glass matrix. As a results, the multiplication of shear bands prevail in the glass matrix, as shown in Fig. 5(c).

\section{Conclusion}

In the current study, quasi-static and dynamic deformation behaviors of an in-situ Ti-based BMG composite were investigated in SLR. The present BMG composite exhibits a homogenous viscous flow under quasi-static deformation, but shear-band-dominated inhomogeneous plastic deformation upon dynamic loading. Strain hardening can be seen in the later deformation stage of the present BMG composite at $713 \mathrm{~K}$ and $2 \times 10^{-4} / \mathrm{s}$ as well as $5 \times 10^{-4} / \mathrm{s}$, which can be ascribed to the nanocrystallization in the glass matrix facilitated by the temperature and low strain rates. The flow stress decreases in both quasi-static and dynamic condition with the increase of the temperature. Due to the effective plastic accommodation of dendrites to the glass matrix, characteristic of the formation of 
shear steps and multiple dislocations, a remarkable plasticity (about $9 \%$ ) has been obtained in the present BMG composite in SLR. Based on the Sellars-Tegart model, the constitutive equation for the present BMG composite upon quasi-static loading is obtained as: $\dot{\varepsilon}=1.53 \times 10^{24}[\sinh (0.00287 \sigma)]^{4.032} \exp \left(-\frac{4.37 \times 10^{5}}{8.3145 T}\right) \cdot$ In addition, the flow stress approximately follows a linear relationship with the temperature: $\sigma \approx 2,791-2.11 T$ upon dynamic loading.

\section{Acknowledgements}

J.S. Li thanks the Natural Science Basic Research Plan in Shaanxi Province of China (2014JM6234), Specialized Research Fund for Doctoral Program of Higher Education (20136102120007), and Program of Introducing Talents of Discipline to Universities (B08040). P. K. Liaw would like to acknowledge the Departement of (DOE), Office of Fossil Energy, National Energy Technology Laboratory(DE-FE-0008855, DE-FE-0024054, and DE- FE-0011194), with Mr. V. Cedro, Mr. R. Dunst and Dr. J.Mullen and also very thanks the support from the National Science Foundation (CMMI-1100080) with the program director, Dr. C. Cooper. J. Bai thanks for the Chinese Scholarship Council (CSC).

\section{Reference}

[1] H. Li, C. Fan, K. Tao, H. Choo, P.K. Liaw, Adv. Mater. 18 (2006) 752-754.

[2] J.C. Qiao, J.M. Pelletier, J. Mater. Sci. Technol. 30 (2014) 523-545.

[3] X.L. Fu, M.J. Tan, Y.S. Wang, A.E.W. Jarfors, M. Gupta, J. Alloys Compd. 549 (2013) 100-104.

[4] B. Yang, M.L. Morrison, P.K. Liaw, R.A. Buchanan, G. Wang, C.T. Liu, M. Denda, Appl. Phys. Lett. 86 (2005) 141904.

[5] J.C. Qiao, S. Cardinal, J.M. Pelletier, H. Kato, J. Alloys Compd. 628 (2015) 357-363.

[6] J.C. Qiao, Y.-J. Wang, J.M. Pelletier, L.M. Keer, M.E. Fine, Y. Yao, Acta Mater. 98 (2015) 43-50.

[7] D.C. Hofmann, J.Y. Suh, A. Wiest, G. Duan, M.L. Lind, M.D. Demetriou, W. Johnson, Nature 451 (2008) 1085-1089.

[8] J.W. Qiao, S. Wang, Y. Zhang, P.K. Liaw, G.L. Chen, Appl. Phys. Lett. 94 (2009) 151905.

[9] C.C. Hays, C.P. Kim, W.L. Johnson, Phys. Rev. Lett. 84 (2000) 2901-2904.

[10] J.W. Qiao, A.C. Sun, E.W. Huang, Y. Zhang, P.K. Liaw, C.P. Chuang, Acta Mater. 59 (2011) 4126-4137.

[11] Z.H. Chu, G.Y. Yuan, H. Kato, G.Q. Xie, D.R. Yan, J. Alloys Compd. 612 (2014) 10-15.

[12] J. Bai, J.S. Li, J. Wang, H.C. Kou, P.K. Liaw, Mater. Sci. Eng. A 627 (2015) 21-26. 
[13] D.C. Hofmann, J.Y. Suh, A. Wiest, M.L. Lind, M.D. Demetriou, W.L. Johnson, Proc. Natl. Acad. Sci. 105 (2008) 20136-20140.

[14] C. Jeon, C.P. Kim, S.H. Joo, H.S. Kim, S. Lee, Acta Mater. 61 (2013) 3012-3026.

[15] P. Gargarella, S. Pauly, M.S. Khoshkhoo, C.S. Kiminami, U. Kühn, J. Eckert, J. Alloys Compd. 663 (2016) 531-539.

[16] J.L. Cheng, G. Chen, C.T. Liu, Y. Li, Sci. Rep. 3 (2013) 2097.

[17] S. Pauly, S. Gorantla, G. Wang, U. Kühn, J. Eckert, Nature Mater. (2010).

[18] Y.S. Wang, G.J. Hao, Y. Zhang, J.W. Qiao, J.P. Lin, Intermetallics 67 (2015) 121-126.

[19] J. Bai, H.C. Kou, J. Wang, J.S. Li, R. Hu, Mater. Lett. 117 (2014) 228-230.

[20] J.W. Qiao, Y. Zhang, H.L. Jia, H.J. Yang, P.K. Liaw, B.S. Xu, Appl. Phys. Lett. 100 (2012) 121902.

[21] J. Cui, J. Li, J. Wang, L. Li, H. Kou, Mater. Des. 90 (2016) 595-600.

[22] K.S. Lee, S. Kim, K.R. Lim, S.H. Hong, K.B. Kim, Y.S. Na, J. Alloys. Compd. 663 (2016) $270-278$.

[23] J. Bai, J.S. Li, J. Wang, J. Cui, L.Y. Li, H.C. Kou, P.K. Liaw, J. Alloys Compd. 639 (2015) $131-138$.

[24] Y.S. Wang, G.J. Hao, R. Ma, Y. Zhang, J.P. Lin, Z.H. Wang, J.W. Qiao, Intermetallics 60 (2015) $66-71$.

[25] M.Y. Chu, Z.M. Jiao, R.F. Wu, Z.H. Wang, H.J. Yang, Y.S. Wang, J.W. Qiao, J. Alloys Compd. 640 (2015) 305-310.

[26] X.L. Fu, Y. Li, C.A. Schuh, J. Mater. Res. 22 (2007) 1564-1573.

[27] X.Y. Zhang, Z.Z. Yuan, X.L. Feng, L.Z. Cui, D.X. Li, Mater. Sci. Eng. A 620 (2015) 352-358.

[28] W.D. Liu, K.X. Liu, J. Appl. Phys. 108 (2010) 033511.

[29] Y. Huang, J. Shen, Y. Sun, J. Sun, J.J.J. Chen, J. Alloys Compd. 504, Supplement 1 (2010) S82-S85.

[30] J.N. Mei, J.L. Soubeyroux, J.J. Blandin, J.S. Li, H.C. Kou, H.Z. Fu, L. Zhou, Intermetallics 19 (2011) 48-53.

[31] W.J. Kim, D.S. Ma, H.G. Jeong, Scr. Mater. 49 (2003) 1067-1073.

[32] M. Bletry, P. Guyot, Y. Brechet, J.J. Blandin, J.L. Soubeyroux, Mater. Sci. Eng., A 387-389 (2004) 1005-1011. 
[33] C. Sellars, W. McTegart, Acta Metall. 14 (1966) 1136-1138.

[34] B. Gun, K.J. Laws, M. Ferry, J. Non-Cryst. Solids 352 (2006) 3896-3902.

[35] C.M. Sellars, W.J. McTegart, Acta Metall. 14 (1966) 1136-1138.

[36] H. Chen, H. Kato, A. Inoue, J. Saida, N. Nishiyama, Appl. Phys. Lett. 79 (2001) 60-62.

[37] G. Neumann, C. Tuijn, in, Pergamon, Amsterdam ;, 2009.

[38] J. Lu, G. Ravichandran, W.L. Johnson, Acta Mater. 51 (2003) 3429-3443.

[39] K.B. Kim, J. Das, F. Baier, J. Eckert, Appl. Phys. Lett. 86 (2005) 171909.

[40] X.L. Fu, M.J. Tan, Y. Chen, A.E.W. Jarfors, M. Gupta, C.H. Shek, Mater. Sci. Eng. A 621 (2015)

1-7.

[41] P.S. Singh, R.L. Narayan, I. Sen, D.C. Hofmann, U. Ramamurty, Mater. Sci. Eng. A 534 (2012) 476-484.

[42] T.G. Nieh, J. Wadsworth, C.T. Liu, T. Ohkubo, Y. Hirotsu, Acta Mater. 49 (2001) 2887-2896.

[43] D.-G. Lee, Y.G. Kim, B. Hwang, S. Lee, Y.T. Lee, Mater. Sci. Eng. A 472 (2008) 316-323. 


\section{Figure captions}

Fig. 1 (a) Microstructure of the as-casted Ti-based BMG composite and (b) The X-ray diffraction pattern, revealing that the present BMG composite consists of the body-centered cubic dendrites and amorphous matrix.

Fig. 2 True stress-strain curves of the present BMG composites at different temperature under quasi-static deformation with various strain rates.

Fig . 3 Variation of the flow stress with various strain rates at different temperature under quasi-static deformation.

Fig. 4 (a) True stress-strain curves of the present BMG composites at different temperature upon dynamic loading. (b) The variation of the flow stress and strain as a function of temperature.

Fig. 5 (a) Macroscopic morphology of the sample after deformation at the strain rate of $2 \times 10^{-4} / \mathrm{s}$ and $683 \mathrm{~K}$ (b) The corresponding microstructure evolution after deformation. (c) Lateral surface after dynamic loading at the strain rate of $1.3 \times 10^{3} / \mathrm{s}$ at $683 \mathrm{~K}$. The magnified images of (c) shown in the inset (c1), clearly demonstrating the formation of multiple shear bands on the lateral surface. (d) The corresponding fracture surface after dynamic loading.

Fig. 6 DSC curves of the samples deformed at the strain rate of $2 \times 10^{-4} / \mathrm{s}$ at $663 \mathrm{~K}$, and $713 \mathrm{~K}$, respectively.

Fig. 7 (a) Plots of $\ln (\dot{\varepsilon})$ as a function of $\ln [\sinh (\alpha \sigma)]$, where the slope of each data set gives n. (b ) Plot of Zener-Hollomon parameter $(\ln Z)$ as a function of $\ln [\sinh (\alpha \sigma)]$, indicating the availability of the Sellars-Tegart model in the present BMG composites.

Fig. 8 Master curve of normalized viscosity $\left(\eta / \eta_{N}\right)$ as a function of $Z$

Fig. 9 The relationship between the flow stress and temperature upon dynamic loading. The solid line 
gives the linear fitting results.

Fig. 10 TEM images of the deformed microstructure upon dynamic loading at $713 \mathrm{~K}$, which shows the formation of shear steps and multiple dislocations (b) HRTEM image of the interface between the dendrites and glass matrix. (b1) and (b2) corresponding to IFFT of the regions marked by black and white rectangular regions, respectively.

Fig. 11 Comparison of the mechanical properties of the present BMG composites together with some other BMGs and composites upon quasi-static (a) and dynamic (b) deformation

Table 1 The calculated parameters of the Sellars-Tegart model for the present BMG composites 

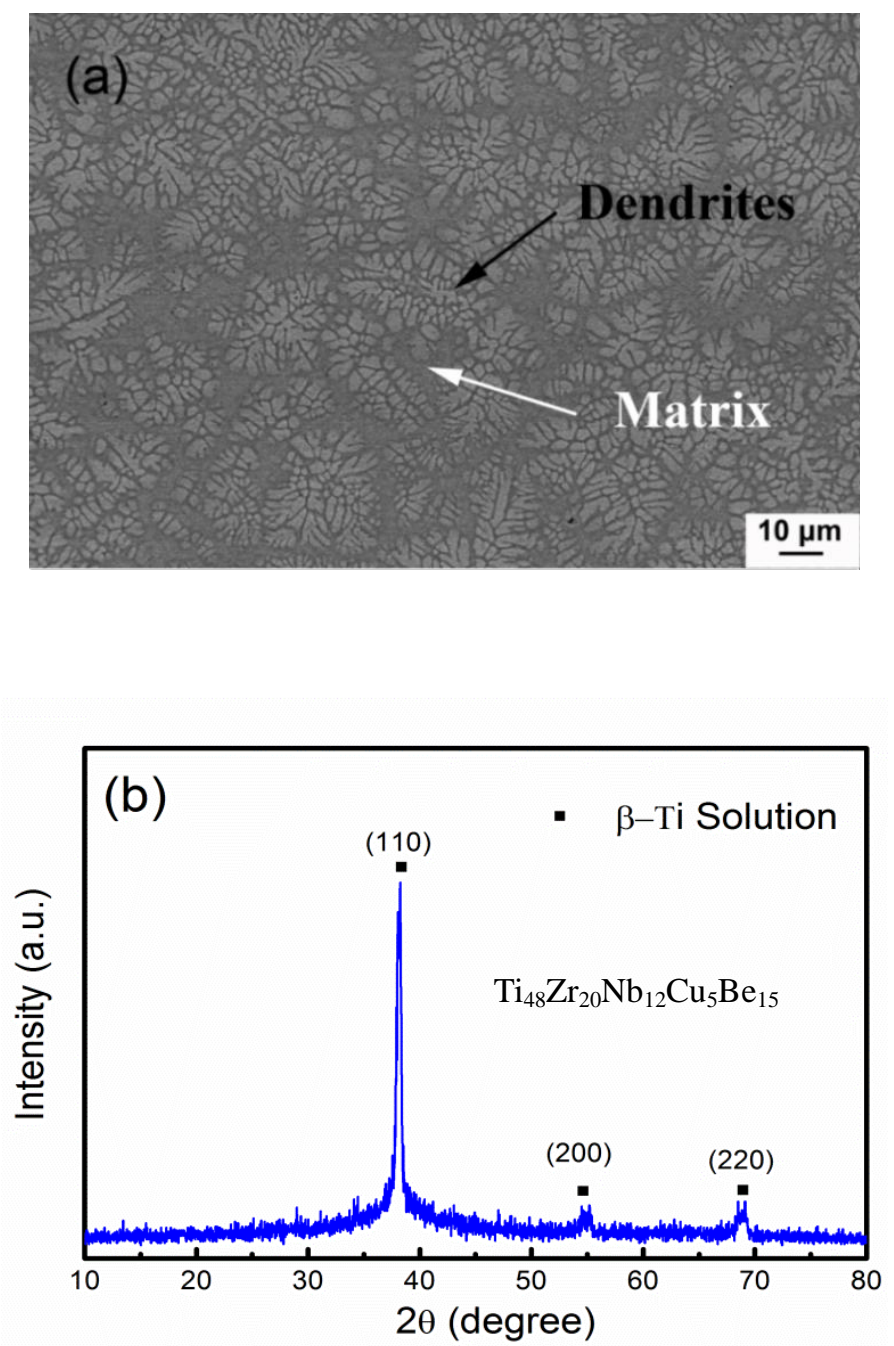

Fig. 1(a) Microstructure of as-casted Ti-based BMG composite. (b) The X-ray diffraction pattern, revealing that the present BMG composite consists of the body-centered cubic dendrites and amorphous matrix. 

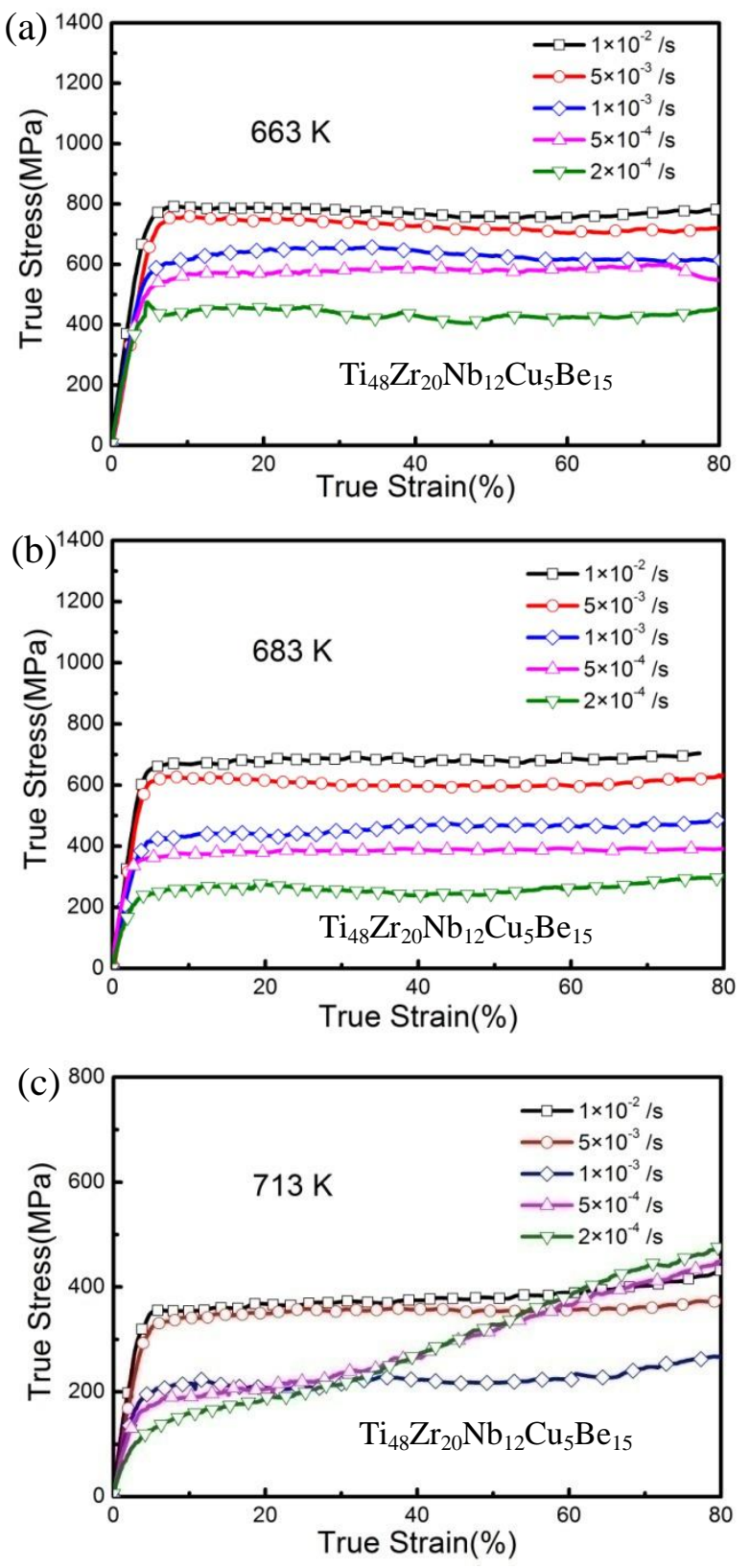

Fig. 2 The true stress-strain curves of the present BMG composites at different temperature upon quasi-static loading with various strain rates. 


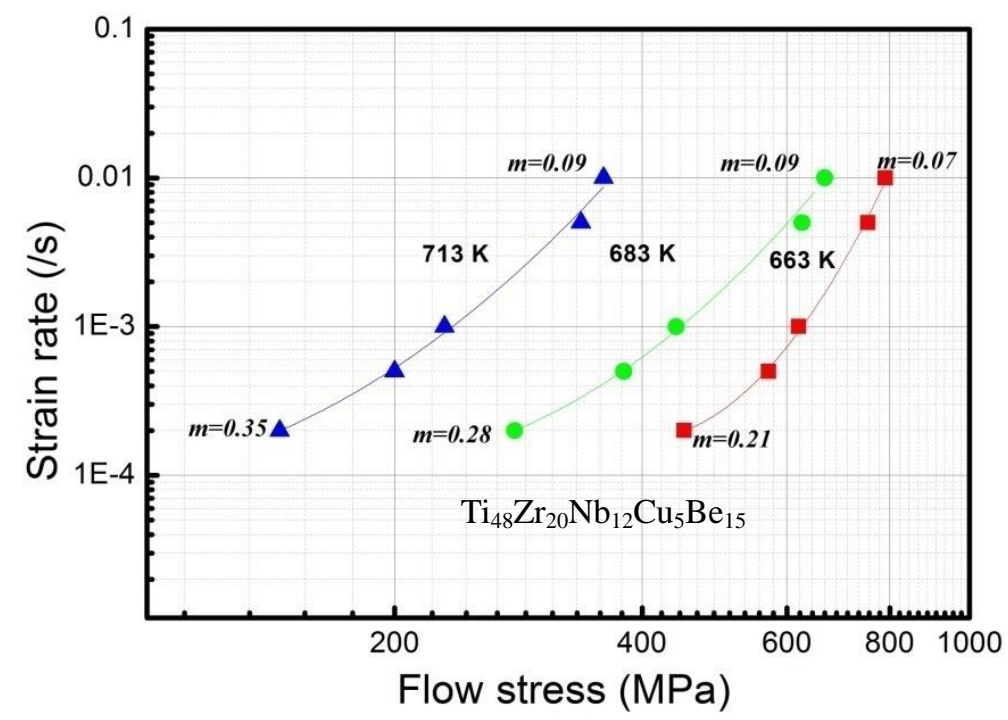

Fig. 3 The variation of the flow stress with various strain rates at different temperature under quasi-static deformation 

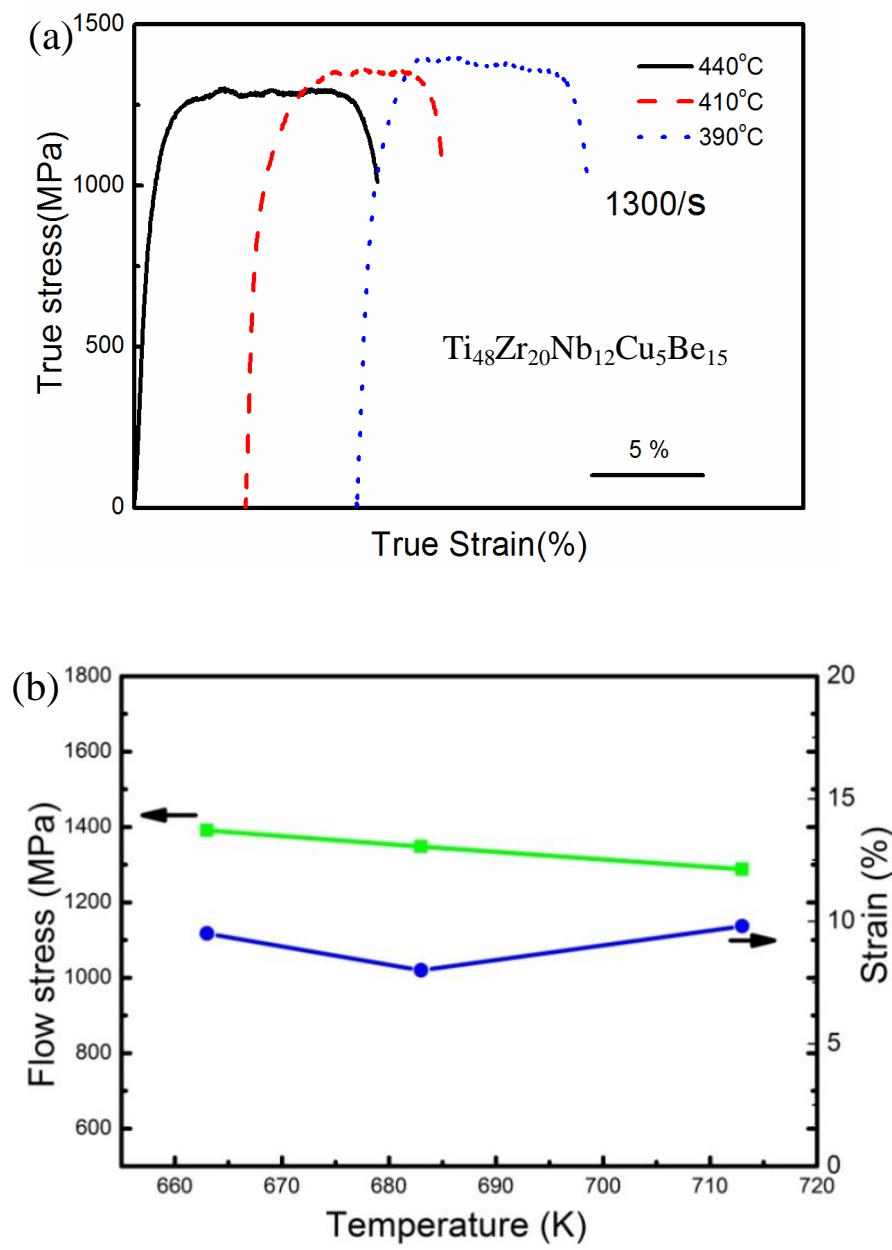

Fig. 4 (a) The true stress-strain curves of the present BMG composites at different temperature upon dynamic loading. (b) The variation of the flow stress and strain as a function of temperature. 

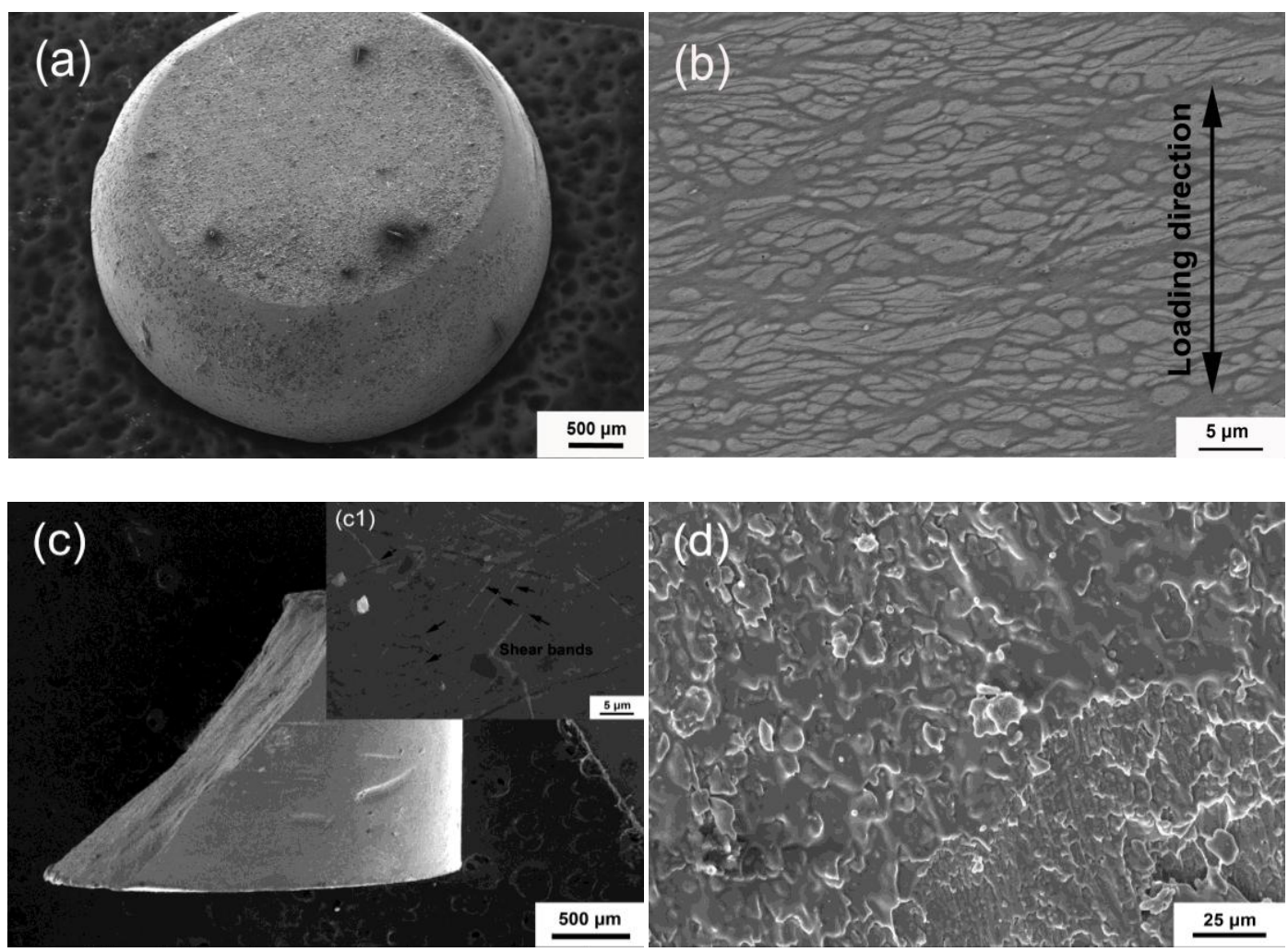

Fig. 5 (a) Macroscopic morphology of the sample after deformation at the strain rate of $2 \times 10^{-4} / \mathrm{s}$ and $683 \mathrm{~K}$ (b) The corresponding microstructure evolution after deformation. (c) Lateral surface after dynamic loading at the strain rate of $1.3 \times 10^{3} / \mathrm{s}$ and $683 \mathrm{~K}$. The magnified images of (c) shown in the inset (c1), clearly demonstrating the formation of multiple shear bands on the lateral surface. (d) The corresponding fracture surface after dynamic loading. 


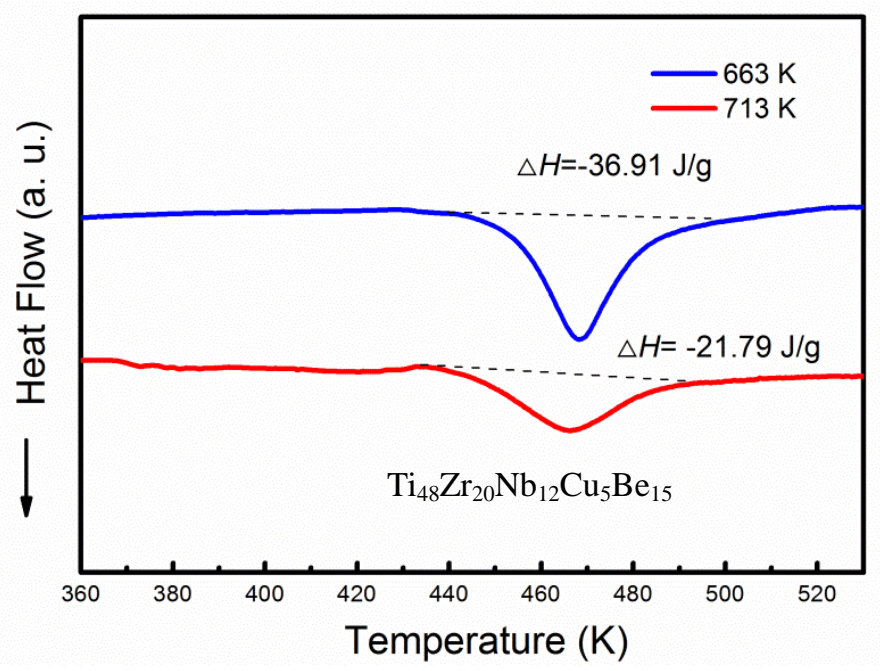

Fig. 6 DSC curves of the samples deformed at the strain rate of $2 \times 10^{-4} / \mathrm{s}, 663 \mathrm{~K}$, and $713 \mathrm{~K}$, respectively. 

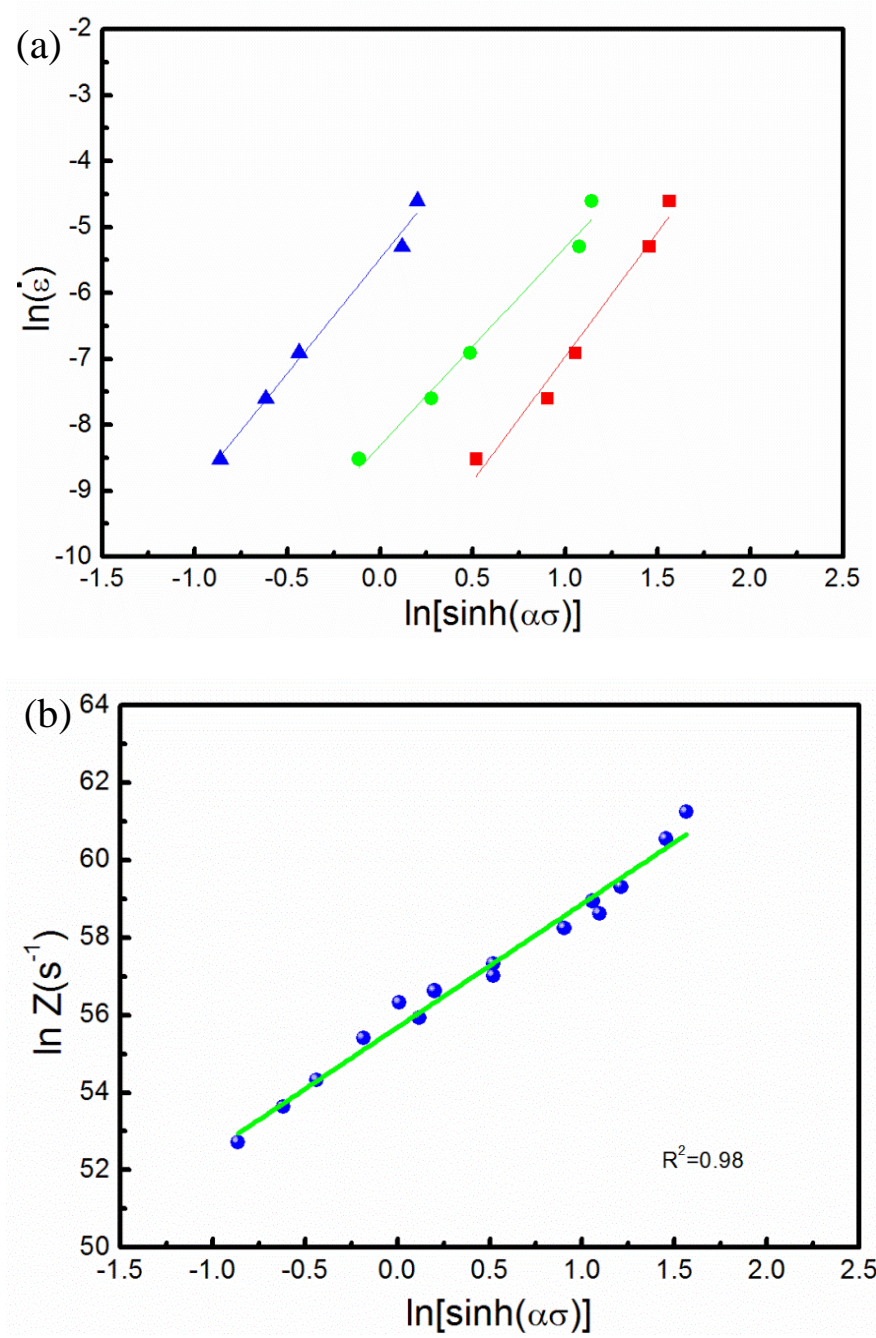

Fig. 7 (a) Plots of $\ln (\dot{\varepsilon})$ as a function of $\ln [\sinh (\alpha \sigma)]$, where the slope of each data set gives n. (b ) Plot of the Zener-Hollomon parameter $(\ln Z)$ as a function of $\ln [\sinh (\alpha \sigma)]$, indicating the availability of the Sellars-Tegart model in the present BMG composites. 


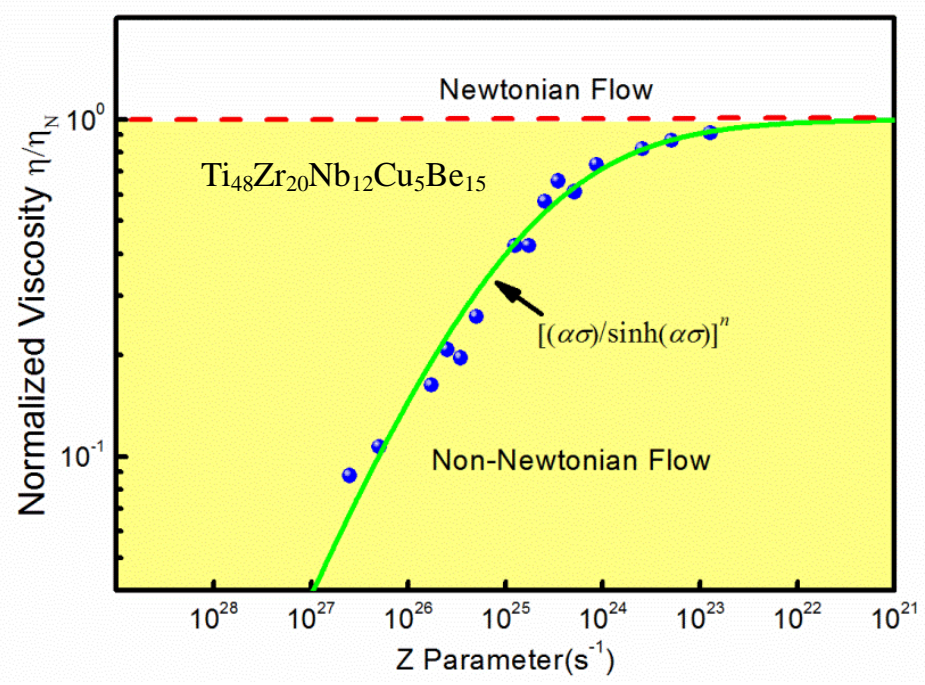

Fig. 8 Master curve of normalized viscosity $\left(\eta / \eta_{N}\right)$ as a function of $Z$ 


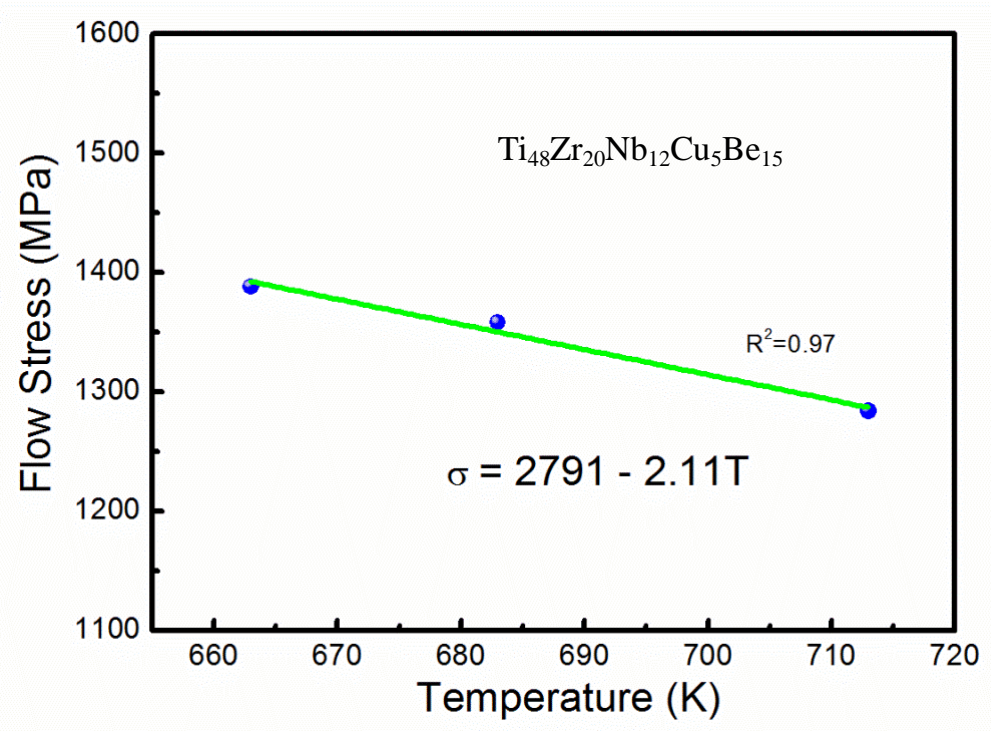

Fig. 9 The relationship between the flow stress and temperature upon dynamic loading. The solid line gives the linear fitting results. 


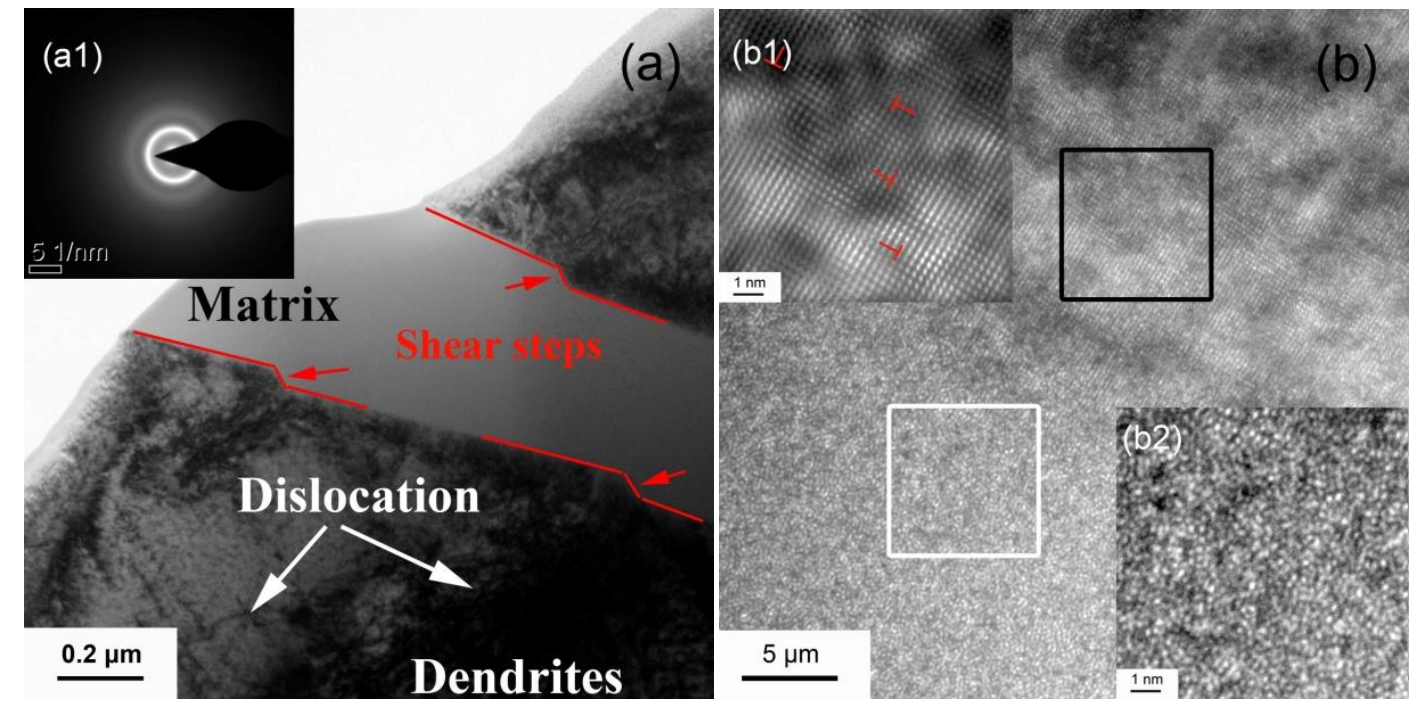

Fig. 10 TEM images of the deformed microstructure upon dynamic loading at $713 \mathrm{~K}$, which shows the formation of shear steps and multiple dislocations (b) HRTEM image of the interface between the dendrites and glass matrix. (b1) and (b2) corresponding to IFFT of the regions marked by black and white rectangular regions, respectively. 

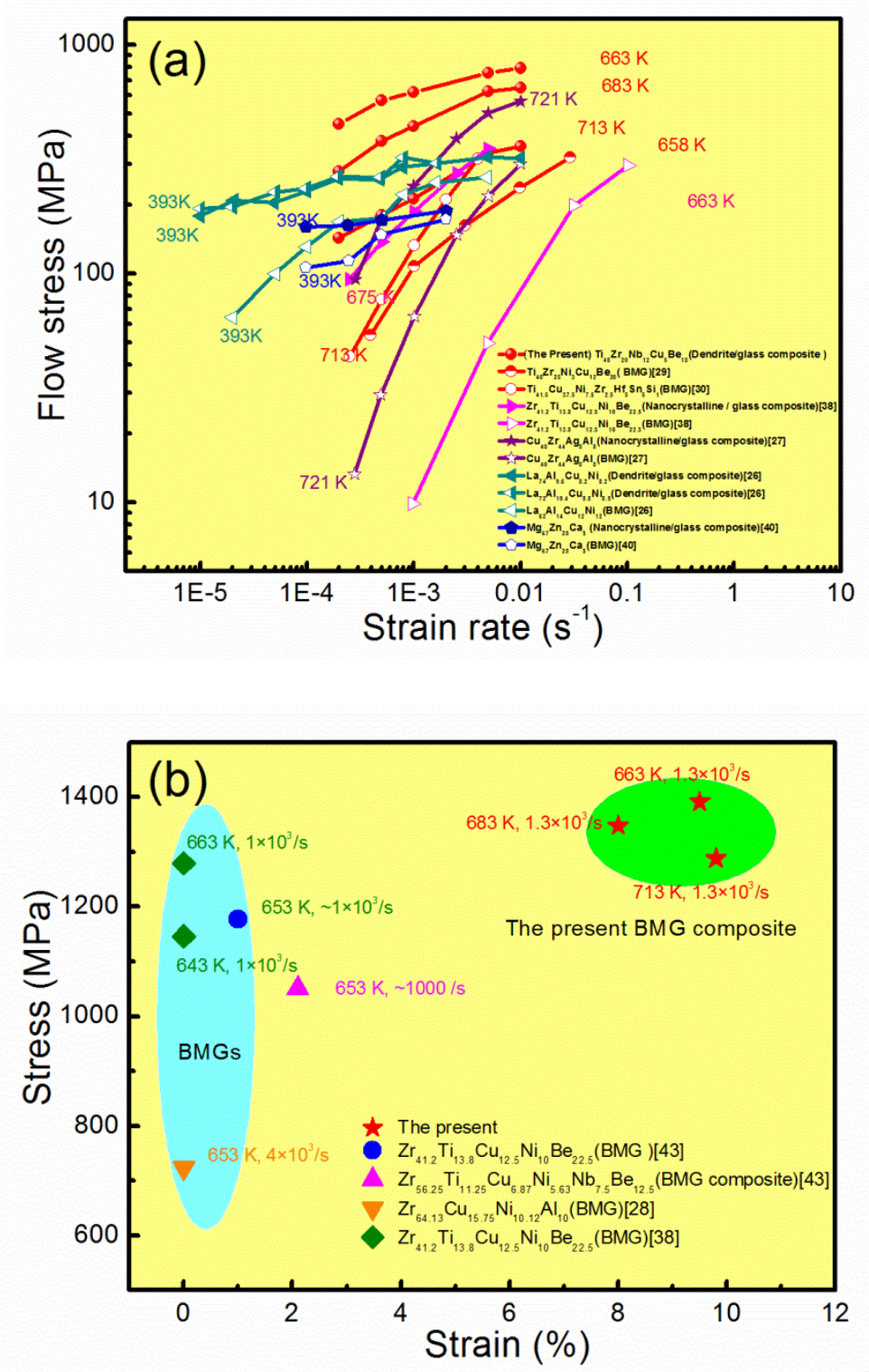

Fig. 11 Comparison of the mechanical properties of the present BMG composites together with some other BMGs and composites upon quasi-static (a) and dynamic (b) deformation. 
Table 1 The calculated parameters of Sellars-Tegart model for the present BMG composites

\begin{tabular}{lcccc}
\hline Parameters & $\mathrm{A}\left(\mathrm{s}^{-1}\right)$ & $\alpha\left(\mathrm{MPa}^{-1}\right)$ & $\mathrm{n}$ & $\mathrm{Q}(\mathrm{KJ} / \mathrm{mol})$ \\
\hline $\mathrm{Ti}_{48} \mathrm{Zr}_{20} \mathrm{Cu}_{5} \mathrm{Nb}_{12} \mathrm{Be}_{15}$ & $1.53 \times 10^{24}$ & 0.00287 & 4.032 & 436.88 \\
\hline
\end{tabular}

